\title{
Proposal of a New Scalar Gauge in Relativity Field Equation
}

\author{
Young Hwan Yun*, Kiho Jang \\ Zero Theoretical Physics Laboratory, Seoul, Republic of Korea \\ Email: *yhyun690@naver.com
}

How to cite this paper: Yun, Y.H. and Jang, K. (2021) Proposal of a New Scalar Gauge in Relativity Field Equation. Journal of High Energy Physics, Gravitation and Cosmology, 7, 1524-1534.

https://doi.org/10.4236/jhepgc.2021.74093

Received: September 15, 2021

Accepted: October 26, 2021

Published: October 29, 2021

Copyright (c) 2021 by author(s) and Scientific Research Publishing Inc. This work is licensed under the Creative Commons Attribution International License (CC BY 4.0).

http://creativecommons.org/licenses/by/4.0/

\begin{abstract}
In this paper we propose a new gauge term in addition to the conventional gauge to acquire complete solution for the linear approximated gravitational equation. The calculation to make general form for the linear gravitational equation uses the well-known Nöether's theorem saying that gauge symmetry is equal to conservation law. The unsolved coefficients in the equation require another condition which is leading to new gauge term. This proposed new gauge is a tensor product by a scalar quantity with a metric tensor having the trace value of 2 . The scalar component in the 5th row and column of Kaluza-Klein's metric tensor can be found as 2 diagonal components in our proposed $4 \times 4$ metric tensor. We also show that only a constant scalar gauge can be allowed in the curved space-time although arbitrary gauge can exist in the linear space-time.
\end{abstract}

\section{Keywords}

Radion, Gravity, Kaluza-Klein Theory, Gauge Symmetry, Relativity

\section{Introduction}

Long years ago, Kaluza and Klein proposed the initial unification theory of the electromagnetic force and gravitational force with their revolutionary 5-dimentional metric tensor. The first metric tensor proposed by Kaluza has a $5 \times 5$ structure and has a scalar component, $k$, in the $5^{\text {th }}$ row and $5^{\text {th }}$ column as can be seen in $(1-1)[1][2]$.

$$
g_{\mu \nu}=\left(\begin{array}{ccccc}
g_{00} & g_{01} & g_{02} & g_{03} & k A_{0} \\
g_{10} & g_{11} & g_{12} & g_{13} & k A_{1} \\
g_{20} & g_{21} & g_{22} & g_{23} & k A_{2} \\
g_{30} & g_{31} & g_{32} & g_{33} & k A_{3} \\
k A_{0} & k A_{1} & k A_{2} & k A_{3} & k
\end{array}\right)
$$


But unfortunately, above tensor does not satisfy the tensor condition, so Klein supplemented and modified it as can be seen in (1-2) [2] [3]. There still exists a scalar component, $k$, in the position of the $5^{\text {th }}$ row and $5^{\text {th }}$ column. The scalar term $k$ is called radion or dilaton or graviscalar by the following theorists [1] [3].

$$
g_{\mu v}=\left(\begin{array}{ccccc}
g_{00}+k A_{0} A_{0} & g_{01}+k A_{0} A_{1} & g_{02}+k A_{0} A_{2} & g_{03}+k A_{0} A_{3} & k A_{0} \\
g_{01}+k A_{0} A_{1} & g_{11}+k A_{1} A_{1} & g_{12}+k A_{1} A_{2} & g_{13}+k A_{1} A_{3} & k A_{1} \\
g_{02}+k A_{0} A_{2} & g_{12}+k A_{1} A_{2} & g_{22}+k A_{2} A_{2} & g_{23}+k A_{2} A_{3} & k A_{2} \\
g_{03}+k A_{0} A_{3} & g_{13}+k A_{1} A_{3} & g_{23}+k A_{2} A_{3} & g_{33}+k A_{3} A_{3} & k A_{3} \\
k A_{0} & k A_{1} & k A_{2} & k A_{3} & k
\end{array}\right)
$$

Differently from the Kaluza-Klein theory, we have shown recently that gravity and electromagnetic force can be unified by using a gauge even with a $4 \times 4$ metric tensor [4] [5]. However, there is no gauge term with scalar component like " $k$ " in the Kaluza-Klein metric tensor. Although the scalar term does not directly affect the electromagnetic field, we have wondered if a scalar gauge can exist even in a $4 \times 4$ metric tensor and have tried to find it.

As can be shown later in this paper, although the gauge in the linear approximated space-time has degree of freedom, there is some restriction in curved space-time. For that reason, we first develop our derivation in the linear approximated gravitational equation rather than the general gravitational equation below.

$$
R^{\mu v}-\frac{1}{2} g^{\mu v} R=\frac{8 \pi G}{c^{4}} T^{\mu v}
$$

In case of $g^{\mu v}=\eta^{\mu v}+k h^{\mu v}$ and $k \ll 1$. The linear approximated equation of (1-3) is acquired like followings [6],

$\partial_{\lambda} \partial^{\lambda} h^{\mu v}+\partial^{\mu} \partial^{v} h-\left(\partial_{\lambda} \partial^{v} h^{\mu \lambda}+\partial_{\lambda} \partial^{\mu} h^{\nu \lambda}\right)-\eta^{\mu v} \partial_{\lambda} \partial^{\lambda} h+\eta^{\mu v} \partial_{\lambda} \partial_{\sigma} h^{\lambda \sigma}=\frac{8 \pi G}{c^{4}} T^{\mu v}$

We go through with a mathematical deployment to see if this equation has a scalar gauge by using Nöether's theorem [7] [8]. Since $T^{\mu v}$ on the right side of the Equation (1-3) is an energy-momentum tensor, the symmetry of the 4 vectors is the symmetry of space-time, and also is the conservation of an energy-momentum tensor $T^{\mu v}$.

In the next chapters, we will first find out a field equation by using Nöether's theorem [7] [8] (gauge symmetry and conservation) and conservation law in general electromagnetic fields, and then extend to the gravitational field with a similar and consistent methodology.

\section{Case of Electromagnetic Equation}

The only four-vectors linear in $A^{v}$ that can be constructed out of $A^{v}$ and no more than two of the differential operators $\partial^{\mu}$ are listed in (2-1) [6].

$$
\partial_{\mu} \partial^{\mu} A^{v}, \partial^{v} \partial_{\mu} A^{\mu}, A^{v}
$$

The general equation using combination of these can be constructed as follows, 


$$
\partial_{\mu} \partial^{\mu} A^{v}+\boldsymbol{a} \partial^{v} \partial_{\mu} A^{\mu}+\boldsymbol{b} A^{v}=4 \pi j^{v}
$$

where $A^{v}$ is rank-1 field tensor, $j^{v}$ is the 4-vector source term (such as charge-current term), and $\boldsymbol{a}, \boldsymbol{b}$ are unknown coefficients.

Its gauge transformation is described as following,

$$
A^{v} \rightarrow A^{v}+\partial^{v} \Lambda
$$

where $\Lambda$ is the gauge term of rank-1 tensor $A^{v}$.

If Equation (2-2) is symmetric with respect to the gauge transformation Equation (2-3), the result of the gauge equation must be 0 like following,

$$
\partial_{\mu} \partial^{\mu} \partial^{v} \Lambda+\boldsymbol{a} \partial^{v} \partial_{\mu} \partial^{\mu} \Lambda+\boldsymbol{b} \partial^{v} \Lambda=0
$$

or

$$
(1+\boldsymbol{a}) \partial^{v} \partial_{\mu} \partial^{\mu} \Lambda+\boldsymbol{b} \partial^{v} \Lambda=0
$$

In order for Equation (2-5) to always hold, the following conditions are necessary.

$$
\begin{gathered}
1+\boldsymbol{a}=0 \quad(\text { i.e. } \quad \boldsymbol{a}=-1) \\
\boldsymbol{b}=0
\end{gathered}
$$

Then Equation (2-2) becomes

$$
\partial_{\mu} \partial^{\mu} A^{v}-\partial^{v} \partial_{\mu} A^{\mu}=4 \pi j^{v}
$$

In another method, if we apply the conservation of energy-momentum $\left(\partial_{v} j^{v}=0\right)$ to Equation (2-2), we have

$$
\partial_{v} \partial_{\mu} \partial^{\mu} A^{v}+\boldsymbol{a} \partial_{v} \partial^{v} \partial_{\mu} A^{\mu}+\boldsymbol{b} \partial_{v} A^{v}=4 \pi \partial_{v} j^{v}=0
$$

or

$$
(1+\boldsymbol{a}) \partial_{v} \partial^{v} \partial_{\mu} A^{\mu}+\boldsymbol{b} \partial_{v} A^{v}=0
$$

By the properties of dummy index, we used followings

$$
\begin{aligned}
& \partial_{\mu} \partial^{\mu}=\partial_{v} \partial^{v} \\
& \partial_{\mu} A^{\mu}=\partial_{\nu} A^{v}
\end{aligned}
$$

From Equation (2-10), we obtain $\boldsymbol{a}=-1$ and $\boldsymbol{b}=0$. This is the same as the result of the gauge symmetry condition. From above results, we can conclude that the gauge symmetry and energy-momentum conservation are the necessary and sufficient conditions in the rank-1 tensor equation, which is consistent with Nöether's theorem. This rank-1 tensor field equation turns out to be the Maxwell's electro-magnetic equation. In next chapter, we can extend to the $2^{\text {nd }}$ rank tensor linear approximated gravitational equation.

\section{Case of Linear Approximated Gravitational Equation}

As we have done with the electromagnetic field, we can proceed with a similar 
procedure in the linear approximated gravitational equation.

In (3-1), we can write down all possible symmetric tensors that can be constructed out of $h^{\mu v}$ and no more than two gradients $\partial^{\sigma}$ which are linear in $h^{\mu v}[6]$.

$$
\partial_{\lambda} \partial^{\lambda} h^{\mu \nu}, \partial^{\mu} \partial^{v} h, \partial_{\lambda} \partial^{v} h^{\mu \lambda}+\partial_{\lambda} \partial^{\mu} h^{\nu \lambda}, \eta^{\mu v} \partial_{\lambda} \partial^{\lambda} h, \eta^{\mu v} \partial_{\lambda} \partial_{\sigma} h^{\lambda \sigma}, h^{\mu v}, \eta^{\mu v} h
$$

By combining these, we can create a generalized equation as shown below.

$$
\begin{aligned}
& \partial_{\lambda} \partial^{\lambda} h^{\mu \nu}+\boldsymbol{a} \partial^{\mu} \partial^{v} h+\boldsymbol{b}\left(\partial_{\lambda} \partial^{v} h^{\mu \lambda}+\partial_{\lambda} \partial^{\mu} h^{\nu \lambda}\right)+\boldsymbol{c} \eta^{\mu v} \partial_{\lambda} \partial^{\lambda} h \\
& +\boldsymbol{d} \eta^{\mu \nu} \partial_{\lambda} \partial_{\sigma} h^{\lambda \sigma}+\boldsymbol{e} h^{\mu \nu}+\boldsymbol{f} \eta^{\mu \nu} h=\frac{8 \pi G}{c^{4}} T^{\mu \nu}
\end{aligned}
$$

where $h^{\mu \nu}$ is rank-2 field tensor, $T^{\mu \nu}$ is the rank-2 source tensor (energymomentum tensor), and $\boldsymbol{a}, \boldsymbol{b}, \boldsymbol{c}, \boldsymbol{d}, \boldsymbol{e}, \boldsymbol{f}$ are unsolved coefficients.

Its gauge transformation can be described as following [6] [9].

$$
h^{\mu v} \rightarrow h^{\mu v}+\partial^{v} \Lambda^{\mu}+\partial^{\mu} \Lambda^{v}
$$

where $\partial^{v} \Lambda^{\mu}+\partial^{\mu} \Lambda^{v}$ is the gauge term of rank-2 tensor $h^{\mu v}$. From now on, we will call this gauge a vector gauge. We name a vector gauge because the gauge is made from vector.

We have referenced the previous trial to find the unknown coefficients, and the following 3 sequential conditions (titled Old-1,2,3) were used in that reference [6].

Old-1. The term $\boldsymbol{e} h^{\mu v}+\boldsymbol{f} \eta^{\mu v} h$ must vanish empirically because it reduces the potential exponentially as the distance increases, so that $\boldsymbol{e}=\boldsymbol{f}=0$.

Old-2. The results that $\boldsymbol{a}=-\boldsymbol{c}, \boldsymbol{b}=-1, \boldsymbol{d}=1, \boldsymbol{e}=0$, and $\boldsymbol{f}=0$ are acquired by using the conservation condition $\left(\partial_{\mu} T^{\mu \nu}=0\right)$.

Old-3. To find the unsolved $\boldsymbol{a}$ or $\boldsymbol{c}$, they tried to replace $h^{\mu v}$ to $h^{\mu \nu}+C \eta^{\mu \nu} h$ (where $\boldsymbol{C}$ is constant) in Equation (3-1) and then acquired $\boldsymbol{a}=1, \boldsymbol{c}=-1$ even if $\boldsymbol{C}=0$.

But Old-3 step is somewhat weird and need more explanation [6].

In the other way, we try to obtain exact solutions using 2 different conditions (titled New-1,2), gauge symmetry and energy-momentum conservation, which is similar to the work in chapter 2 .

New-1. Gauge symmetry condition:

The linear equation of rank-2 tensor of $h^{\mu \nu}$ and $T^{\mu \nu}$, as the energy momentum source, is given by Equation (2-1). To see the gauge symmetry, we try to apply $\partial^{v} \Lambda^{\mu}+\partial^{\mu} \Lambda^{v}$. To reduce the calculation, we only put the gauge term into Equation (3-2). Then the result becomes zero like (3-4).

$$
\begin{aligned}
& \partial_{\lambda} \partial^{\lambda}\left(\partial^{v} \Lambda^{\mu}+\partial^{\mu} \Lambda^{v}\right)+\boldsymbol{a} \partial^{\mu} \partial^{v}\left(\partial_{\sigma} \Lambda^{\sigma}+\partial_{\lambda} \Lambda^{\lambda}\right)+\boldsymbol{b}\left(\partial_{\lambda} \partial^{v}\left(\partial^{\mu} \Lambda^{\lambda}+\partial^{\lambda} \Lambda^{\mu}\right)\right. \\
& \left.+\partial_{\lambda} \partial^{\mu}\left(\partial^{v} \Lambda^{\lambda}+\partial^{\lambda} \Lambda^{v}\right)\right)+\boldsymbol{c} \eta^{\mu v} \partial_{\lambda} \partial^{\lambda}\left(\partial_{\sigma} \Lambda^{\sigma}+\partial_{\lambda} \Lambda^{\lambda}\right)+\boldsymbol{d} \eta^{\mu \nu} \partial_{\lambda} \partial_{\sigma}\left(\partial^{\lambda} \Lambda^{\sigma}\right. \\
& \left.+\partial^{\sigma} \Lambda^{\lambda}\right)+\boldsymbol{e}\left(\partial^{v} \Lambda^{\mu}+\partial^{\mu} \Lambda^{v}\right)+\boldsymbol{f} \eta^{\mu v}\left(\partial_{\sigma} \Lambda^{\sigma}+\partial_{\lambda} \Lambda^{\lambda}\right)=0
\end{aligned}
$$

Multiplying both sides by $\eta_{\mu v}$ 


$$
\begin{aligned}
& \eta_{\mu v} \partial_{\lambda} \partial^{\lambda}\left(\partial^{v} \Lambda^{\mu}+\partial^{\mu} \Lambda^{v}\right)+\boldsymbol{a} \eta_{\mu v} \partial^{\mu} \partial^{v}\left(\partial_{\sigma} \Lambda^{\sigma}+\partial_{\lambda} \Lambda^{\lambda}\right) \\
& +\boldsymbol{b} \eta_{\mu v}\left(\partial_{\lambda} \partial^{v}\left(\partial^{\mu} \Lambda^{\lambda}+\partial^{\lambda} \Lambda^{\mu}\right)+\partial_{\lambda} \partial^{\mu}\left(\partial^{v} \Lambda^{\lambda}+\partial^{\lambda} \Lambda^{v}\right)\right) \\
& +4 \boldsymbol{c} \partial_{\lambda} \partial^{\lambda}\left(\partial_{\sigma} \Lambda^{\sigma}+\partial_{\lambda} \Lambda^{\lambda}\right)+4 \boldsymbol{d} \partial_{\lambda} \partial_{\sigma}\left(\partial^{\lambda} \Lambda^{\sigma}+\partial^{\sigma} \Lambda^{\lambda}\right) \\
& +4 \boldsymbol{e}\left(\partial_{\mu} \Lambda^{\mu}+\partial_{v} \Lambda^{v}\right)+4 \boldsymbol{f}\left(\partial_{\sigma} \Lambda^{\sigma}+\partial_{\lambda} \Lambda^{\lambda}\right)=0
\end{aligned}
$$

The last two terms on the left side are similar terms according to the following properties.

$$
\partial_{\mu} \Lambda^{\mu}+\partial_{v} \Lambda^{v}=\partial_{\sigma} \Lambda^{\sigma}+\partial_{\lambda} \Lambda^{\lambda}
$$

In this case, the following conditions are required for Equation (3-5) to hold always.

$$
\begin{gathered}
1+\boldsymbol{b}=0 \\
\boldsymbol{a}+\boldsymbol{b}=0 \\
c+d=0 \\
\boldsymbol{e}+\boldsymbol{f}=0
\end{gathered}
$$

Unlike electromagnetic equation, in gravity, $\boldsymbol{e}$ and $\boldsymbol{f}$ (the coefficients of non- derivative terms) are alive even if the gauge symmetry is satisfied. From Equation (3-7) to Equation (3-10), we have that $\boldsymbol{b}=-1$ and $\boldsymbol{a}=1$, but $\boldsymbol{c}, \boldsymbol{d}, \boldsymbol{e}$ and $\boldsymbol{f}$ remain unknown. This means that all the coefficients are not determined by only $\partial^{v} \Lambda^{\mu}+\partial^{\mu} \Lambda^{v}$. For the gravitational equation, unlike the electromagnetic equation, the gauge symmetry does not guarantee the conservation of energy-momentum.

New-2. Energy-momentum conservation:

When we apply the condition for conservation of energy-momentum $\left(\partial_{\mu} T^{\mu v}=0\right)$ to Equation (3-2), then

$$
\begin{aligned}
& \partial_{\mu} \partial_{\lambda} \partial^{\lambda} h^{\mu \nu}+\boldsymbol{a} \partial_{\mu} \partial^{\mu} \partial^{v} h+\boldsymbol{b} \partial_{\mu}\left(\partial_{\lambda} \partial^{v} h^{\mu \lambda}+\partial_{\lambda} \partial^{\mu} h^{v \lambda}\right)+\boldsymbol{c} \partial^{v} \partial_{\lambda} \partial^{\lambda} h \\
& +\boldsymbol{d} \partial^{v} \partial_{\lambda} \partial_{\sigma} h^{\lambda \sigma}+\boldsymbol{e} \partial_{\mu} h^{\mu v}+\boldsymbol{f} \eta^{\mu v} \partial_{\mu} h=\frac{8 \pi G}{c^{4}} \partial_{\mu} T^{\mu v}=0
\end{aligned}
$$

Notice that similar terms have the same colors. In order for Equation (3-11) to always hold, the following conditions are necessary.

$$
\begin{gathered}
1+\boldsymbol{b}=0 \\
\boldsymbol{a}+\boldsymbol{c}=0 \\
\boldsymbol{b}+\boldsymbol{d}=0 \\
\boldsymbol{e} \partial_{\mu} h^{\mu v}+\boldsymbol{f} \eta^{\mu v} \partial_{\mu} h=0
\end{gathered}
$$

Therefore

$$
\begin{gathered}
\boldsymbol{b}=-1 \\
\boldsymbol{a}=-\boldsymbol{c} \\
\boldsymbol{d}=1
\end{gathered}
$$

Since $\partial_{\mu} h^{\mu v}$ and $\eta^{\mu v} \partial_{\mu} h$ are different terms, Equation (3-11) can be established by setting both $\boldsymbol{e}=0$ and $\boldsymbol{f}=0$. But in the case of Equation (3-15), we 
treat the 2 terms like a similar term, because when $\partial_{\mu} h^{\mu \nu}$ and $\eta^{\mu v} \partial_{\mu} h$ are multiplied by $\eta_{\mu v}$, both become terms of $h$. For this reason there is a possibility other than $\boldsymbol{e}=\boldsymbol{f}=0$.

According to Equations (3-16)-(3-18), a,c,e and $\boldsymbol{f}$ are still unknown.

This looks that Nöether's theorem saying gauge symmetry is conservation, does not hold. Because we do not know all the variables yet, we need more condition to find out the unknown variables. To obtain additional equations, the step Old-3 uses the fact that the field equation should be maintained even if the trace reversed form like (3-19) is used [6]. But Old-3 calculation looks a little weird, and we show that calculation is inconsistent. After that we suggest the way to resolve the inconsistency.

At first, let's define h-bar like (3-19).

$$
\bar{h}^{\mu v}=h^{\mu v}+C \eta^{\mu v} h \quad(\text { where } C \text { is arbitrary) }
$$

If we substitute Equation (3-19) into Equation (3-2), then we have

$$
\begin{aligned}
& \partial_{\lambda} \partial^{\lambda}\left(h^{\mu v}+\boldsymbol{C} \eta^{\mu v} h\right)+\boldsymbol{a} \partial^{\mu} \partial^{v}(h+4 \boldsymbol{C} h) \\
& -\left(\partial_{\lambda} \partial^{v}\left(h^{\lambda \mu}+\boldsymbol{C} \eta^{\lambda \mu} h\right)+\partial_{\lambda} \partial^{\mu}\left(h^{\lambda v}+\boldsymbol{C} \eta^{\lambda v} h\right)\right) \\
& -\boldsymbol{a} \eta^{\mu v} \partial_{\lambda} \partial^{\lambda}(h+4 \boldsymbol{C} h)+\eta^{\mu v} \partial_{\lambda} \partial_{\sigma}\left(h^{\lambda \sigma}+\boldsymbol{C} \eta^{\lambda \sigma} h\right)=\frac{8 \pi G}{c^{4}} T^{\mu v}
\end{aligned}
$$

Separating the terms of $h^{\mu v}$ and $C \eta^{\mu \nu} h$

$$
\begin{aligned}
& \partial_{\lambda} \partial^{\lambda}\left(h^{\mu v}\right)+\boldsymbol{a} \partial^{\mu} \partial^{v}(h)-\left(\partial_{\lambda} \partial^{v}\left(h^{\lambda \mu}\right)+\partial_{\lambda} \partial^{\mu}\left(h^{\lambda v}\right)\right)-\boldsymbol{a} \eta^{\mu v} \partial_{\lambda} \partial^{\lambda}(h) \\
& +\eta^{\mu \nu} \partial_{\lambda} \partial_{\sigma}\left(h^{\lambda \sigma}\right)+\boldsymbol{C}\left(-\partial_{\lambda} \partial^{v}\left(\eta^{\lambda \mu} h\right)-\boldsymbol{a} \partial^{\mu} \partial^{v}(4 h)+\partial_{\lambda} \partial^{\lambda}\left(\eta^{\mu \nu} h\right)\right. \\
& \left.+\boldsymbol{a} \eta^{\mu \nu} \partial_{\lambda} \partial^{\lambda}(4 h)-\eta^{\mu v} \partial_{\lambda} \partial_{\sigma}\left(h^{\lambda \sigma}\right)\right)=\frac{8 \pi G}{c^{4}} T^{\mu v}
\end{aligned}
$$

Rearranging

$$
\begin{aligned}
& \partial_{\lambda} \partial^{\lambda}\left(h^{\mu v}\right)+\boldsymbol{a} \partial^{\mu} \partial^{v}(h)-\left(\partial_{\lambda} \partial^{v}\left(h^{\lambda \mu}\right)+\partial_{\lambda} \partial^{\mu}\left(h^{\lambda v}\right)\right)-\boldsymbol{a} \eta^{\mu v} \partial_{\lambda} \partial^{\lambda}(h) \\
& +\eta^{\mu v} \partial_{\lambda} \partial_{\sigma}\left(h^{\lambda \sigma}\right)-\partial_{\lambda} \partial^{v}\left(C \eta^{\lambda \mu} h\right)-\boldsymbol{a} \partial^{\mu} \partial^{v}(C 4 h)+C\left(\partial^{\mu} \partial^{v} h+\partial^{\mu} \partial^{v} h\right) \\
& +\boldsymbol{a} \boldsymbol{C} \eta^{\mu v} \partial_{\lambda} \partial^{\lambda}(4 h)-\boldsymbol{C} \eta^{\mu v} \partial_{\lambda} \partial_{\sigma} h=\frac{8 \pi G}{c^{4}} T^{\mu v}
\end{aligned}
$$

The same color is a similar term. When the blue term is defined as $\boldsymbol{a}^{\prime}$, then

$$
\boldsymbol{a}^{\prime}=\boldsymbol{a}(1-4 C)+2 \boldsymbol{C}
$$

or

$$
C=\frac{a^{\prime}-a}{2-4 a}
$$

On the other hand, the green term in Equation (3-22) should disappear so the coefficients should be 0 as shown below.

$$
4 C a-2 C=0
$$

or

$$
2 C(-2 a+1)=0
$$


The solution of Equation (3-26) is either $\boldsymbol{C}=0$ or $\boldsymbol{a}=1 / 2$. But because $\boldsymbol{a}=1 / 2$ cannot maintain the field equation, $\boldsymbol{C}$ must be 0 , and $\boldsymbol{a}^{\prime}=\boldsymbol{a}$ in Equation (3-24). Even though $\boldsymbol{a}$ cannot be determined in this approach, $\boldsymbol{a}$ and $\boldsymbol{a}^{\prime}$ are set to 1 without justifiable explanation. The requirement is to find the value of $\boldsymbol{a}$. But choosing $\boldsymbol{C}=0$ doesn't satisfy the initial request of independent $C$. In the next chapter, we suggest other way to prevent this contradiction.

\section{Derivation of Scalar Gauge}

In Equation (3-26), in order for $\boldsymbol{a}$ to be an integer, the value of trace should be 2, instead of 4 (i.e. $\eta_{\mu \nu} \eta^{\mu v}=2$ ). Then, Equation (3-23) becomes

$$
a^{\prime}=a(1-2 C)+2 C
$$

Comparing Equation (4-1) with Equation (3-23), 4 is changed to 2. $\boldsymbol{a}^{\prime}$ must be equal to $\boldsymbol{a}$ to maintain the field equation form, thus we have

$$
\boldsymbol{a}^{\prime}=\boldsymbol{a}(1-2 \boldsymbol{C})+2 \boldsymbol{C}=\boldsymbol{a}
$$

Rearranging

$$
0=-2 a C+2 C=2 C(1-a)
$$

For equation (4-3) to hold independent of $\boldsymbol{C}, \boldsymbol{a}$ must be 1 . This is what we hope for. In Equation (3-19), $h$ plays as only a scalar, so we can generalize it and change $h$ to a general scalar $\Lambda$. Therefore, we propose a new gauge as shown below and try to acquire all the unknown coefficients.

$$
h^{\mu v} \rightarrow h^{\mu v}+\eta^{\prime \mu v} \Lambda
$$

where $\Lambda$ is an arbitrary scalar and $\eta^{\prime \mu \nu}$ is a metric tensor with trace value 2. From now on, we call this gauge a scalar gauge compared with a vector gauge in chapter 3. We replace $h^{\mu v}$ with $\eta^{\prime \mu v} \Lambda$ in Equation (3-2) and this gauge equation should be 0 .

$$
\begin{aligned}
& \partial_{\lambda} \partial^{\lambda} \eta^{\prime \mu v} \Lambda+\boldsymbol{a} \partial^{\mu} \partial^{v}(2 \Lambda)+\boldsymbol{b}\left(\partial_{\lambda} \partial^{v} \eta^{\prime \mu \lambda} \Lambda+\partial_{\lambda} \partial^{\mu} \eta^{\prime \nu \lambda} \Lambda\right)+\boldsymbol{c} \eta^{\mu v} \partial_{\lambda} \partial^{\lambda}(2 \Lambda) \\
& +\boldsymbol{d} \eta^{\mu \nu} \partial_{\lambda} \partial_{\sigma} \eta^{\prime \sigma \lambda} \Lambda+\boldsymbol{e} \eta^{\prime \mu v} \Lambda+\boldsymbol{f} \eta^{\mu v}(2 \Lambda)=0
\end{aligned}
$$

Multiplying both sides by $\eta_{\mu v}^{\prime}$ and using the properties for $\eta_{\mu \nu}^{\prime} \eta^{\prime \mu v}=2$, $\eta_{\mu \nu}^{\prime} \eta^{\mu \nu}=2$, we obtain

$$
\begin{aligned}
& \partial_{\lambda} \partial^{\lambda} 2 \Lambda+\boldsymbol{a} \eta_{\mu \nu}^{\prime} \partial^{\mu} \partial^{\nu}(2 \Lambda)+\boldsymbol{b}\left(\partial_{\lambda} \partial^{\nu} 2 \Lambda+\partial_{\lambda} \partial^{\mu} 2 \Lambda\right)+2 \boldsymbol{c} \partial_{\lambda} \partial^{\lambda}(2 \Lambda) \\
& +2 \boldsymbol{d} \partial_{\lambda} \partial_{\sigma} \eta^{\prime \sigma \lambda} \Lambda+2 \boldsymbol{e} \Lambda+2 \boldsymbol{f}(2 \Lambda)=0
\end{aligned}
$$

Similar terms have the same colors. Rearranging Equation (4-6),

$$
2(1+2 \boldsymbol{c}+\boldsymbol{d}) \partial_{\lambda} \partial^{\lambda} \Lambda+(2 \boldsymbol{a}+2 \boldsymbol{b}) \partial^{\mu} \partial^{\nu} \eta_{\mu \nu}^{\prime} \Lambda+2(\boldsymbol{e}+2 \boldsymbol{f}) \Lambda=0
$$

The following conditions are necessary for the above expression to hold always.

$$
\begin{gathered}
1+2 c+d=0 \\
a+b=0 \\
e+2 \boldsymbol{f}=0
\end{gathered}
$$


As we can see Equation (4-8)-(4-10), coefficients $\boldsymbol{a}, \boldsymbol{b}, \boldsymbol{c}, \boldsymbol{d}, \boldsymbol{e}$ and $\boldsymbol{f}$ are not obtained by $\eta^{\prime \mu v} \Lambda$ only. When we use two gauge conditions for the vector and the scalar gauge, we use Equation (3-7)-(3-10) and Equation (4-8)-(4-10), thus all coefficients can be found like (4-11).

$$
\boldsymbol{a}=1, \boldsymbol{b}=-1, \boldsymbol{c}=-1, \boldsymbol{d}=1, \boldsymbol{e}=\boldsymbol{f}=0
$$

This is the purpose of our trial for searching an additional new gauge named a scalar gauge. Applying Equation (4-11) to Equation (3-2), we have

$\partial_{\lambda} \partial^{\lambda} h^{\mu v}+\partial^{\mu} \partial^{v} h-\left(\partial_{\lambda} \partial^{v} h^{\mu \lambda}+\partial_{\lambda} \partial^{\mu} h^{\nu \lambda}\right)-\eta^{\mu v} \partial_{\lambda} \partial^{\lambda} h+\eta^{\mu v} \partial_{\lambda} \partial_{\sigma} h^{\lambda \sigma}=\frac{8 \pi G}{c^{4}} T^{\mu v}$

We can write the full gauge transformation as follows.

$$
h^{\mu v} \rightarrow h^{\mu v}+\partial^{v} \Lambda^{\mu}+\partial^{\mu} \Lambda^{v}+\eta^{\mu v} \Lambda
$$

In Equation (4-13) $\partial^{v} \Lambda^{\mu}+\partial^{\mu} \Lambda^{v}$ is fully $4 \times 4$ form, on the other hand $\eta^{\prime \mu v} \Lambda$ is only a $2 \times 2$ form included in the $4 \times 4$ equation. The equations of coefficients that can be obtained through gauge symmetry and conservation can be summarized in Table 1.

It was shown that all coefficients were determined using case (1) and case (2). This shows that symmetry is conservation, which is equal to case (3). The combination for case (1), (2), (3) is left for the our future study.

\section{Gauge Symmetry of Scalar Gauge in Curved Space-Time}

In this chapter, we want to see whether the scalar gauge obtained in the previous chapter is also symmetric in the curved space-time like the case of approximated gravitational equation by putting it into the Bianchi identities which represent the curved space-time equations.

First, let's check the Bianchi identity.

$$
R_{i j k l}+R_{i k l j}+R_{i l j k}=0
$$

Here $R_{i j k l}$ is written as follows [10].

$$
R_{i j k l}=\partial_{k} \Gamma_{i j l}-\partial_{l} \Gamma_{i j k}+\Gamma_{j l}^{p} \Gamma_{i p k}-\Gamma_{j k}^{p} \Gamma_{i p l}
$$

\begin{tabular}{|c|c|c|c|c|}
\hline Case & Case (1): vector gauge & Case (2): scalar gauge & Case (3): conservation & \\
\hline Conditions & $h^{\mu v} \rightarrow h^{\mu v}+\partial^{v} \Lambda^{\mu}+\partial^{\mu} \Lambda^{v}$ & $h^{\mu v} \rightarrow h^{\mu v}+\eta^{\prime \mu v} \Lambda$ & $\partial_{\mu} T^{\mu v}=0$ & \\
\hline \multirow{4}{*}{ Relation between coefficients } & $1+b=0$ & $1+2 c+d=0$ & $1+b=0$ & $(3-12)$ \\
\hline & $a+b=0 \quad(3-8)$ & $a+b=0$ & $a+c=0$ & $(3-13)$ \\
\hline & $c+d=0 \quad(3-9)$ & $e+2 f=0 \quad(4-10)$ & $b+d=0$ & $(3-14)$ \\
\hline & $e+f=0 \quad(3-10)$ & & $e \partial_{\mu} h^{\mu v}+f \eta^{\mu v} \partial_{\mu} h=0$ & $(3-15)$ \\
\hline Unknown coefficients & $c, d, e, f$ & $a, b, c, d, e, f$ & \multicolumn{2}{|l|}{$a, c, e, f$} \\
\hline
\end{tabular}

Here, $\Lambda$ is very small, so if we ignore the $3^{\text {rd }}$ and $4^{\text {th }}$ on the right side of Equation (5-2),

Table 1. Coefficients relations for each case. 


$$
\begin{gathered}
R_{i j k l} \approx \partial_{k} \Gamma_{i j l}-\partial_{l} \Gamma_{i j k} \\
=\partial_{k}\left(\partial_{j} g_{i l}+\partial_{l} g_{i j}-\partial_{i} g_{j l}\right)-\partial_{l}\left(\partial_{j} g_{i k}+\partial_{k} g_{i j}-\partial_{i} g_{j k}\right)
\end{gathered}
$$

The gauge to be substituted here is as follows.

$$
g^{\mu v} \rightarrow g^{\mu v}+\eta^{\prime \mu v} \Lambda
$$

Let's substitute the gauge term only and check if there is symmetry.

$$
\begin{aligned}
& R_{i j k l}=\partial_{k}\left(\eta_{i l}^{\prime} \partial_{j} \Lambda+\eta_{i j}^{\prime} \partial_{l} \Lambda-\eta_{j l}^{\prime} \partial_{i} \Lambda\right)-\partial_{l}\left(\eta_{i k}^{\prime} \partial_{j} \Lambda+\eta_{i j}^{\prime} \partial_{k} \Lambda-\eta_{j k}^{\prime} \partial_{i} \Lambda\right) \\
& R_{i k l j}=\partial_{l}\left(\eta_{i k}^{\prime} \partial_{j} \Lambda+\eta_{i j}^{\prime} \partial_{k} \Lambda-\eta_{j k}^{\prime} \partial_{i} \Lambda\right)-\partial_{j}\left(\eta_{i l}^{\prime} \partial_{k} \Lambda+\eta_{i k}^{\prime} \partial_{l} \Lambda-\eta_{k l}^{\prime} \partial_{i} \Lambda\right) \\
& R_{i l j k}=\partial_{j}\left(\eta_{i k}^{\prime} \partial_{l} \Lambda+\eta_{i l}^{\prime} \partial_{k} \Lambda-\eta_{k l}^{\prime} \partial_{i} \Lambda\right)-\partial_{k}\left(\eta_{i l}^{\prime} \partial_{j} \Lambda+\eta_{i j}^{\prime} \partial_{l} \Lambda-\eta_{j l}^{\prime} \partial_{i} \Lambda\right)
\end{aligned}
$$

It can be seen that they exactly cancel each other and have symmetry. Now let's check the $2^{\text {nd }}$ Bianchi identity [10]

$$
\nabla_{l} R_{\text {mijk }}+\nabla_{j} R_{\text {mikl }}+\nabla_{k} R_{\text {milj }}=0
$$

Substituting Equation (5-5) here

$$
\begin{aligned}
& \nabla_{l} R_{m i j k}=\nabla_{l} \partial_{j}\left(\eta_{m k}^{\prime} \partial_{i} \Lambda+\eta_{m i}^{\prime} \partial_{k} \Lambda-\eta_{k i}^{\prime} \partial_{m} \Lambda\right)-\nabla_{l} \partial_{k}\left(\eta_{m i}^{\prime} \partial_{j} \Lambda+\eta_{m j}^{\prime} \partial_{i} \Lambda-\eta_{j i}^{\prime} \partial_{m} \Lambda\right) \\
& \nabla_{j} R_{m i k l}=\nabla_{j} \partial_{k}\left(\eta_{m l}^{\prime} \partial_{i} \Lambda+\eta_{m i}^{\prime} \partial_{l} \Lambda-\eta_{l i}^{\prime} \partial_{m} \Lambda\right)-\nabla_{j} \partial_{l}\left(\eta_{m i}^{\prime} \partial_{k} \Lambda+\eta_{m k}^{\prime} \partial_{i} \Lambda-\eta_{k i}^{\prime} \partial_{m} \Lambda\right) \\
& \nabla_{k} R_{m i l j}=\nabla_{k} \partial_{l}\left(\eta_{m j}^{\prime} \partial_{i} \Lambda+\eta_{m i}^{\prime} \partial_{j} \Lambda-\eta_{j i}^{\prime} \partial_{m} \Lambda\right)-\nabla_{k} \partial_{j}\left(\eta_{m i}^{\prime} \partial_{l} \Lambda+\eta_{m l}^{\prime} \partial_{i} \Lambda-\eta_{l i}^{\prime} \partial_{m} \Lambda\right)
\end{aligned}
$$

In general, since $\nabla_{l} \partial_{j} \partial_{i} \neq \nabla_{j} \partial_{l} \partial_{k}$, the gauge symmetry does not hold in the above case. However, the special case where $\Lambda=$ constant is possible to satisfy

\begin{tabular}{|c|c|c|c|}
\hline Name & dimension & Space-time & Transformation \\
\hline Kaluza & \multirow{2}{*}{5} & linear approximated/curved & $g_{\mu v} \rightarrow g_{\mu v}+k g_{3 v} A_{v}+k g_{\mu 3} A_{\mu}+g_{44} k$ \\
\hline Klein & & linear approximated/curved & $g_{\mu v} \rightarrow g_{\mu v}+k A_{\mu} A_{v}+k g_{3 v} A_{v}+k g_{\mu 3} A_{\mu}+g_{44} k$ \\
\hline \multirow{2}{*}{ Yun, et al. } & \multirow{2}{*}{4} & linear approximated & $\begin{array}{c}g_{\mu v} \rightarrow g_{\mu v}+\partial_{\mu} A_{v}+\partial_{v} A_{\mu}+\eta_{\mu v}^{\prime} \Lambda \\
\text { however, } \operatorname{tr}\left(\eta_{\mu v}^{\prime}\right)=2\end{array}$ \\
\hline & & curved & $\begin{array}{c}g_{\mu v} \rightarrow g_{\mu \nu}+\nabla_{\mu} A_{v}+\nabla_{v} A_{\mu}+\eta_{\mu v} \Lambda \\
\text { however, } \nabla_{\mu} A_{v}+\nabla_{v} A_{\mu}=0 \text { and } \Lambda \text { : constant }\end{array}$ \\
\hline
\end{tabular}
the gauge symmetry. In other words, only the global gauge has symmetry for the scalar gauge. Since the space is curved, it is considered that the symmetry is more limited than in the case of linear approximation. It is also unusual that there is no such distinction in the Kaluza-klein theory. We summarize all gauge terms in Table 2.

The expression that describes the gauge as the electromagnetic field $A_{\mu}$ in Table 2, refers to previous papers [4] [5]. Even there, space-time is studied dividing by both linear approximated and curved [4] [5].

Table 2. Metric tensor transformation 


\section{Conclusion}

We have revealed that there is a gauge including a scalar quantity even in the four dimensions. We have found that the previously known vector gauge alone can not satisfy the law of conservation of symmetry in the linear approximated gravitational equation, and an additional gauge is needed. So, by introducing a scalar gauge, we could solve this problem completely. In Kaluza-Klein theory, the scalar component compared with the scalar gauge proposed in this paper exists only in the fifth row and column of the five-dimentional metric tensor. But in this paper, we have shown that scalar gauges (trace value of $\eta_{\mu \nu}^{\prime}$ as 2) exist at two points on the diagonal of a metric tensor. It is expected that physical interpretation with respect to the scalar gauge will be studied in the future. It is also found that, although the gauge in the linear approximated space-time has a degree of freedom, the gauge is constant in curved space-time. That is to say, it does not have a local gauge, but a global gauge in curved space-time. It looks natural that space-time is more symmetrical when it is more linear and vice versa. We may conclude that the limit of symmety depends on the limit of curvatue of the space-time and the symmtry term becomes a constant from a variable as space-time becomes curved.

\section{Conflicts of Interest}

The authors declare no conflicts of interest regarding the publication of this paper.

\section{References}

[1] Theodor, K. (1921) Zum Unitätsproblem in der Physik (PDF). Sitzungsberichte der Preussischen Akademie der Wissenschaften (Physikalisch-Mathematische Klasse). PP. 966-972.

[2] Straub, W.O. (2008) Kaluza Klein Theory Pasadena, California. www.physicsforums.com/attachments/kaluza-klein-straub-pdf.55316/

[3] Oskar, K. (1926) Quantentheorie und fünfdimensionale Relativitätstheorie. Zeitschrift für Physik, 37, 895-906. https://doi.org/10.1007/BF01397481

[4] Yun, Y.H., Jang, K. and Sung, Y.K. (2021) Unification of Gravitational and Electromagnetic Fields Using Gauge Symmetry. Journal of High Energy Physics, Gravitation and Cosmology, 7, 344-351. https://doi.org/10.4236/jhepgc.2021.71018

[5] Yun, Y.H. and Jang, K. (2021) Unification of Gravitational and Electromagnetic Fields in Curved Space-Time Using Gauge Symmetry of Bianchi Identities. Journal of High Energy Physics, Gravitation and Cosmology, 7, 1202-1212. https://doi.org/10.4236/jhepgc.2021.73071

[6] Ruffini, O. (1994) Gravitation and Spacetime. 2nd Edition, W. W. Norton \& Company, New York, 134-146.

[7] Emmy, N. (1918) Invariante Variationsprobleme. Nachrichten von der Königliche Gesellschaft der Wissenschaften zu Göttingen, Mathematisch-Physikalische Klasse, 235-257.

[8] Emmy, N. (1971) Invariant Variation Problems. Transport Theory and Statistical 
Physics, 1, 186-207. https://doi.org/10.1080/00411457108231446

[9] Kentaro, Y. (1957) The Theory of Lie Derivatives and Its Applications. Bibliotheca Mathematica 3. Amsterdam, New York, North-Holland.

[10] Su, L.G. (2019) Aha! General Theory of Relativity. YESM Publishing Co. Ltd., Seoul, 74-76. 